\title{
Simulation of Honeybee Nectar Foraging for Determining Effects on Local Flora
}

\author{
$\underline{\text { J. J. García Adeva }}^{\text {a b }}$, L. Lach ${ }^{\text {c d }}$ and M. Reynolds ${ }^{a}$ b

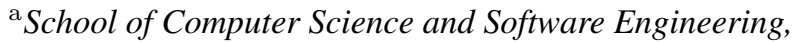 \\ University of Western Australia, Perth WA, Australia \\ ${ }^{\mathrm{b}}$ CRC for National Plant Biosecurity, Canberra ACT, Australia \\ ${ }^{\mathrm{c}}$ School of Plant Biology, University of Western Australia, Perth WA, Australia \\ ${ }^{\mathrm{d}}$ Centre for Integrative Bee Research (CIBER), University of Western Australia, Perth WA, Australia \\ Email: jjgaecsse.uwa.edu.au
}

\begin{abstract}
This work presents a simulator of honeybee (Apis mellifera) colony foraging behavior. Honeybees are social insects that show a high level of organisation and very complex behaviour. This includes the coordination of foraging efforts thanks to their sophisticated mechanism of advertising and recruitment, which includes the well-known waggle dance. This foraging process is needed for general pollination and makes honeybees crucial for a sustainable environment.

There exists previous work in the area of honeybee modelling and simulation. Some simulator models use such a fine resolution that can only run for real-time hours. Other work is focused on simply replicating a published observation. Instead, we are interested in simulating long time periods (e.g. months) and learning how the foraging spread patterns and distributions can affect the local flora or contribute to the transmission of diseases or parasites.
\end{abstract}

The main motivation for this work is to support the decision making process in the Biosecurity context. Biosecurity plays a key role in the economic viability of Australia's plant industries due to the devastating effects that plant pests and diseases can cause. Hence, the importance of preventing the spread of diseases between apiaries or to a disease free area (e.g. Fire Blight (Erwinia amylovora) is a serious disease in many fruits and uses honeybees as one of its main vectors for transmission).

The simulator is Web based ${ }^{1}$ so that users do not have to install or update software, or worry about enough computational power or platform compatibility. The user interface allows the introduction of the simulation parameters (e.g. spatial resolution and size, model configuration settings, visual specification of the simulation environment on the map) and receive a detailed results report. A simulation of several weeks usually takes a few seconds to complete.

The simulation model is stochastic and discrete in time with 1-trip time steps, which is approximated by difference equations. It is also explanatory, meaning that its behavioural rules depend on well-established entomological knowledge. It can support any number of beehives and floral patches over a two dimensional landscape of unrestricted size and varying resolution. The main simulation model has four interrelated models that include i) a spatial model that represents the landscape to simulate by a grid of cells; ii) a flora model that represents a patch with a number of flowers and seasons defined by nectar quality and quantity; iii) a weather model that considers time periods of temperature and daily hours of light; iv) a foraging model, which represents the foraging behaviour of a honeybee colony.

Keywords: simulation modelling, spatio-temporal model, honeybee, foraging, biosecurity management.

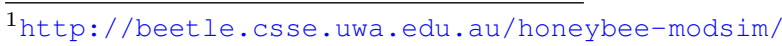




\section{INTRODUCTION}

The ability of honey bee (Apis mellifera) colonies to maximise their foraging efficiency is key to the colonies' continued survival. Individual foragers seek and retrieve pollen and nectar from flowers to feed the colony. Floral patches differ in the quality and quantity of their nectar and pollen, the size of the patch, and the distance from the colony, all factors that affect the profitability of foraging on the patch [Winston, 1991]. The profitability of a floral patch is communicated by individual foragers to other bees in the colony via complex behaviors and other signals. These other individuals may alter their behavior accordingly, and their responses may in turn affect the behavior of the original forager [Seeley, 1985]. Thus foraging in honey bees is a dynamic and highly interactive process that is driven by the sharing of information about the location and quality of resources in the surrounding environment.

The foraging behaviors and hairiness of honeybees mean that they often inadvertently transfer substances, such as pollen, among plants. Honey bees are capable of increasing the yield in $96 \%$ of animal-pollinated crops [Potts et al., 2010], and the value of the increased yield and quality achieved by honey bee pollination of crops in the U.S. is estimated as \$14.6 billion [Morse and Calderone, 2000]. Because honey bees can also act as vectors of some plant diseases such as Fire Blight (Erwinia amylovora) and also as vectors of biological control agents (e.g. the microbe Aureobasidium pullulans, which can be used to attack E. amylovora [Moosbeckhofer et al., 2008]), understanding patterns of foraging behavior will aid in implementing prevention or facilitation measures, respectively.

Incursion and outbreak management are key aspects to biosecurity, which is a major concern in a country like Australia where economic viability of plant industries rely to an extent on freedom from pests and diseases. Strategies to test pre-emptive surveillance and estimate rates of spread of pests or diseases in a spatial environment in real time are not currently available. A 'what if' scenario simulation tool is needed to address this shortcoming [García Adeva et al., 2010]. The aim of this project is to provide such a decision support tool. In consequence, simulating honeybee foraging behaviour will allows us to determine the extent and rate at which surrounding flora may be affected by a disease such as Fire Blight.

The structure of this paper is as follows: in Section 2, we discuss some related work; Section 3 describes the models that define the simulator; Section 4 offers some initial experimental results. We finish with some concluding remarks in Section 5.

\section{RELATED WORK}

There is a significant quantity and variety of research in simulation or modelling of different aspects of honeybees. Some of the most relevant include de Vries and Biesmeijer [1998], which consists of a simulation limited to replicate the foraging experiment that Seeley et al. [1991] performed. Also based on this same experiment, Camazine and Sneyd [1991] proposed a foraging model based on differential equations for foraging, which considers nectar quality in the decision making process for resource abandonment and recruitment of foraging bees. Ghosh and Marshall [2005] proposed a model of learning and collective decision making in honeybees engaged in nectar foraging. However, this model is limited to one of two foraging sites where one site has better nectar than the other in order to understand decision making processes by interacting swarm entities. Bailis et al. [2010] proposes a very well-implemented simulator that models individual foraging bees at a very high level of detail in order to compare the value of sharing food source position information through the waggle dance and private information about previously encountered food sources.

What these previous simulators have in common is that they can only run for short periods of real time (e.g. one day [Camazine and Sneyd, 1991] or even a few hours [Bailis et al., 2010]). Most of them are also fairly restrictive in what type of environment (e.g. only one beehive; a specific number of foraging bees or nectar sources). In contrast, we are interested in much longer periods of time (e.g. months) and, most importantly, to help answer the question about how foraging patterns and distributions can affect the local flora, hence potentially contributing to the transmission of diseases or parasites, or supporting the distribution of certain biological control agents. 


\section{SIMULATION MODEL}

Because traditional mathematical models that provide analytical solutions are only possible for extremely simplified environments, we employ simulation modelling where the abstraction of the particular entity to predict is solved by steps numerically [Swannack, 2008]. The simulation model is based on empirical data and expert knowledge in the field of honeybee ecology and management. Simulation models are often divided between deterministic and stochastic [Odum, 1971]. Deterministic simulation models always produce the same result for a given set of input data and model parameters, whereas stochastic ones include one or more random processes, usually as a way of dealing with uncertainty [Law and Kelton, 1991]. While the flora model (see Section 3.2), weather model (see Section 3.3), and spatial model (see Section 3.1) are deterministic, the foraging model (see Section 3.4) is stochastic.

The evolution of the simulation process is approximated by a difference equation. Being $t$ the duration of the simulation process, we define $\Delta t$ as the time-step used to partition $t$ into so that $y_{n+1}=y_{n}+$ $\Delta t f\left(y_{n}, x_{n}\right)$. In this case, $\Delta t$ corresponds to one foraging trip, with several of these happening within each simulation day. Given the average temperature during the day $T_{i}$ and number of daylight hours $h_{i}$ on day $d_{i}$, the maximum number of foraging trips $f_{i}^{\max }=\mu h_{i}$, with $\mu=0.8$ when $15<T<33, \mu=0$ when $T<8$, or $\mu=0.4$ otherwise. The final actual number of foraging trips will be $f_{i} \leq f_{i}^{\max }$ if the daily nectar is depleted before the end of the day.

The simulation parameters are parsed in order to create the grid representing the simulation area by using the spatial model described in Section 3.1. At the beginning of each simulation day the appropriate average temperature is selected by applying the model described in Section 3.3; then the current daily season of each flower patch is chosen using the model described in Section 3.2. For each foraging trip in the day, the foraging model detailed in Section 3.4 is used to describe the honeybee foraging behaviour.

\subsection{Spatial Model}

The spatial model provides a two-dimensional abstraction of the foraging bees as they spread over an area containing flower patches. The simulation area consists of a landscape whose bounds are initially defined by the user through the simulator's user interface. The extent will usually vary from a few hectares to several hundred thousand $\mathrm{km}^{2}$. The landscape can contain areas (i.e. polygons) representing floral patches and locations indicating beehives.

This landscape is represented as a rectangular planar region $\mathcal{L}$ of width $w_{\mathcal{L}}$ and height $h_{\mathcal{L}}$. Discretisation of $\mathcal{L}$ is performed by means of a rectangular sparse grid $\mathcal{G}$ with its maximum number of horizontal and vertical cells being $w_{\mathcal{G}} \times h_{\mathcal{G}}$. A grid cell is defined by $g=(x, y) \in \mathcal{G}$, where $x, y \in \mathbb{Z} ; 0 \leq x<h_{\mathcal{G}}$; and $0 \leq y<h_{\mathcal{G}}$, with only those non-empty cells being created dynamically when needed. Therefore, $w_{\mathcal{G}}$ and $h_{\mathcal{G}}$ also indicate the simulation resolution, with its maximum value limited by such a cell size where any given cell contains at least 1 flower.

The translation from a coordinate $c=($ lat,lon $) \in \mathcal{L}$ to its cell position $g \in \mathcal{G}$ is calculated with $x=\left\lfloor\mathrm{d}_{\mathrm{h}}(c) w_{\mathcal{G}} w_{\mathcal{L}}\right\rfloor$ and $y=\left\lfloor\mathrm{d}_{\mathrm{v}}(c) h_{\mathcal{G}} h_{\mathcal{L}}\right\rfloor$, where $\mathrm{d}_{\mathrm{v}}(c)$ and $\mathrm{d}_{\mathrm{h}}(c)$ indicate vertical and horizontal distances respectively from $c$ to the bottom-left coordinate of $\mathcal{L}$.

All grid cells are square in shape and have the same size, based on the horizontal resolution selected by the simulator's user (typical values will be $50-500$ ). Because this grid configuration resembles a raster image, there is a plethora of algorithms from the discipline of computer graphics to take advantage from. A cell carries information about how much nectar there is, based on the average number of flowers and the average net nectar per flower. Given an average nectar quantity per flower $q$ and its nectar quality rate $l \in[0,1]$, net nectar is defined as their product.

An area indicating a floral patch is expressed as a closed polygon defined by the list of its ordered (either clockwise or counterclockwise) vertex coordinates $\mathcal{V}_{\mathcal{L}}=\left(v_{0}, v_{1}, v_{2}, \ldots, v_{n}, v_{0}\right)$ with $v_{i}\left(\right.$ lat $_{i}$, lon $\left._{i}\right) \in$ $\mathcal{L}, 0 \leq i \leq n$. Discretising an area $\mathcal{V}_{\mathcal{L}}$ to obtain its corresponding $\mathcal{V}_{\mathcal{G}}$ simply consists of translating each vertex $v_{i} \in \mathcal{L}$ into corresponding cell positions $v_{i} \in \mathcal{G}$. Another instance of a frequently required operation is to determine whether a particular cell is included within an area, which we achieve by applying the Jordan curve theorem as proposed by Haines [1994]. 


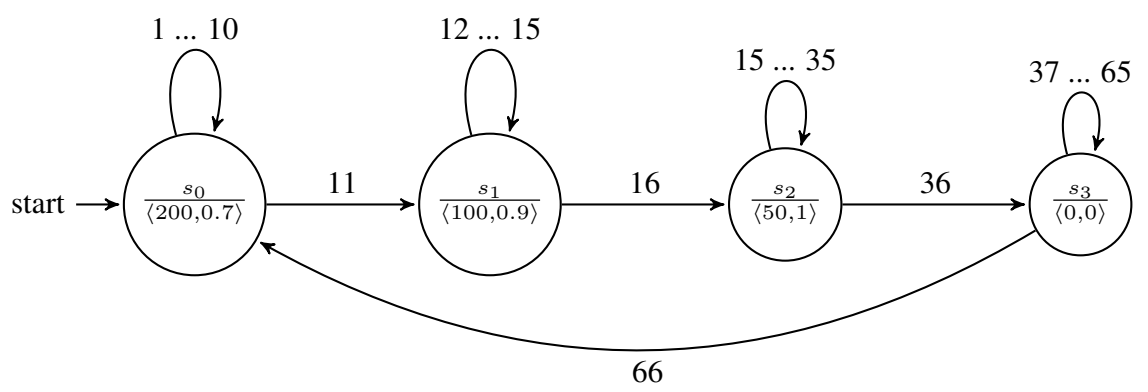

Figure 1: The FSM representing the 65-day cycle of a flower patch with four seasons $\langle 10,200,0.7\rangle$, $\langle 5,100,0.9\rangle,\langle 20,50,1\rangle$, and $\langle 30,0,0\rangle$.

\subsection{Flora Model}

A flower patch is an area containing the following general information: total number of flowers in the area and one or more cyclic seasons. A season indicates for how many days these flowers have the same average quantity and quality of daily nectar production.

A traditional Finite State Machine (FSM) is used to represent a sequence of seasons corresponding to the same flower patch. Considering the set of flower seasons $E=\left\{e_{0}, e_{1}, \ldots, e_{k}\right\}$ each season $e_{i}, 0 \leq i \leq k$ can be described with a three-tuple $\left\langle d_{i}, q_{i}, l_{i}\right\rangle$ where $d_{i}$ expresses the number of season days, while $q_{i}$ indicates the average nectar quantity value per flower during this period and $l_{i} \in[0,1]$ corresponds to the nectar quality rate value. The machine representing these seasons has states $S=\left\{s_{0}, s_{1}, \ldots, s_{k}\right\}$ to represent $E$, hence $|E|=|S|$. The input alphabet includes all the consecutive day numbers for $E$, thus consisting of $I=\{1,2, \ldots, t\}$, where $t=1+\sum_{i=0}^{k} d_{i}$. The output alphabet is given by all the two-tuples extracted from $E$, producing $O=\left\{\left\langle q_{0}, l_{0}^{r}\right\rangle,\left\langle q_{1}, l_{1}\right\rangle, \ldots,\left\langle q_{k}, l_{k}\right\rangle\right\}$. The transition from one machine state to the next occurs when the input for the current state is the last day of the season represented. A transition from the last state (i.e. flowers season) to the first state is automatically created to represent the cyclic nature of seasons. This transition function has to be triggered on each simulation day for all flower areas.

Figure 1 shows the machine representing the four flower patch seasons $\langle 10,200,0.7\rangle,\langle 5,100,0.9\rangle$, $\langle 20,50,1\rangle$, and $\langle 30,0,0\rangle$. This means a total natural cycle of 65 days, thus $t=66$. This corresponds to four states $S=\left\{s_{0}, s_{1}, s_{2}, s_{4}\right\}$, with outputs $O=\{\langle 200,0.7\rangle,\langle 100,0.9\rangle,\langle 50,1\rangle,\langle 0,0\rangle\}$, and inputs $I=\{1,2, \ldots, 66\}$.

\subsection{Weather Model}

The simulator uses weather information that is crucial for determining the number of daily foraging trips. This information includes temperature (in ${ }^{\circ} \mathrm{C}$ ) and number of daylight hours for the whole simulation area. These temperature periods are provided as a sequence of three-tuples, each one specifying a number of days along with the temperature and the number of daylight hours during them such as $\left\langle d_{i}, T_{i}, h_{i}\right\rangle$. The sum of the number of days for all these pairs $\sum_{i=0}^{k} d_{i}$ corresponds to the total number of days of simulation.

Like the Flora Model described in Section 3.2, this model is based on a FSM. In this case, each state $s_{i} \in S$ represents a number of consecutive days with the same temperature $T_{i}$ and daylight hours $h_{i}$. Hence the number of states $|S|$ is determined by the number of temperature periods. The input alphabet includes all the added period days $I=\{0,1,2, \ldots, d\}$. The output $o_{i} \in O$ that corresponds to $s_{i}$ is the temperature and daylight hours for that period $\left\langle T_{i}, h_{i}\right\rangle$. The transition from one state to the next occurs when the input for the current state corresponds to the last day of the period it represents.

\subsection{Foraging Model}

From a given population of forager bees at a beehive, this model aims to determine i) how many foragers will visit particular flowers depending on proximity, nectar quality, and nectar quantity; and ii) the number 


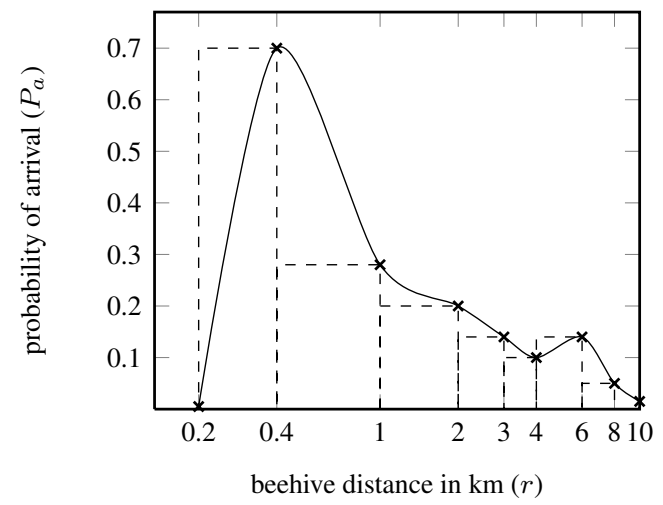

(a) Foraging distances [Seeley, 1985].

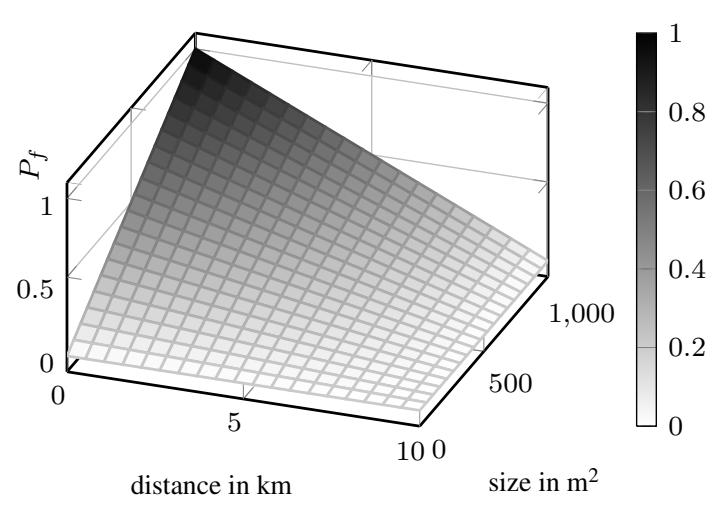

(b) Flower patch detection by size and beehive distance.

Figure 2: Probability distributions of the dispersal kernel used by the foraging model.

of foraging trips for a particular day. The foraging distance and number of individuals finding a flower patch at a certain distance is defined by a kernel, which describes the spatial distribution of foragers in the honeybee vicinity as a function of distance from the beehive and the size of flower patches.

Foraging is similar to local dispersal frequently found in insect modelling. There are many kernel-based dispersal models found in the literature based on distribution such as Laplace, Gaussian, Ribbens [Clark et al., 1998], and Cauchy [Shaw, 1995], which may be considered as arbitrary equations that fit particular dispersal data sets well. Using these kernels require that previously parameters for the chosen distribution to be estimated by registering the dispersal distances between occurrences in distribution data from one time period to the next, so that the distribution can be fitted to the frequency distribution of distances [Pitt, 2008].

Our dispersal kernel, however, uses an empirical probability distribution to determine foraging distances. Figure 2(a) details the probability distribution used to determine how far foragers will fly as reported by Seeley [1985]. For instance, $70 \%$ of bees will forage within $(0.2,0.4] \mathrm{km}, 28 \%$ will do it within $(0.4,1] \mathrm{km}$, and so forth. Figure 2(b) provides the graphical representation of the probability of suitable flower patches being detected by nearby foragers based on distance and size, expressed by $P_{f}=\min \left(\frac{s}{1000}\left(1-\frac{d}{10}\right) \gamma, 1\right)$, where $s \in[0,1000]$ indicates the flower patch size in $\mathrm{m}^{2}, d \in[0,10]$ is the distance in $\mathrm{km}$ between the flower patch and the beehive, and $\gamma>0$ corresponds to the scale parameter, where the larger its value, the higher $P_{f}$.

These two distributions are combined to determine the probability that each cell in a flower patch is visited by honeybees. The basic process for making this decision is based on the grid $\mathcal{G}$, where a cell $g_{i}=$ $\left(x_{i}, y_{i}\right) \in \mathcal{G}$ in this grid represents a discrete location in the simulation area, contained by flower patches $F=\left\{f_{0}, f_{1}, \ldots, f_{m}\right\}$. The foraging bees from a beehive are represented by $B=\left\{b_{0}, b_{1}, \ldots, b_{n}\right\}$. By traversing all the possible dispersal distance ranges shown by Figure 2(a) such as $r_{a}^{b}, r_{b}^{c}, r_{c}^{d}, \ldots$ along with the minimum possible distance increment $\Delta r$ in $\mathcal{G}$ (i.e. the length of a cell side) for as many times as possible (i.e. $\max \left\{n-x_{i}+1, x_{i}, m-y_{i}+1, y_{i}\right\}$ ), we obtain a list of cells $C=\left(c_{0}, c_{1}, \ldots, c_{k}\right.$ ) belonging to the ring that corresponds to the first foraging distances interval $\left[r_{a}^{b}, r_{b}^{c}\right)$. This ring of cells is created by applying the well-known midpoint algorithm [Aken, 1984], thus creating circles of cells as long as $r_{a}^{b} \leq r<r_{b}^{c}$. Those cells from $C$ that are contained by any flower patch in $F$ are selected and ordered according to their probability of being found $P_{f}$, to then be used to sequentially distribute the bees from $B$. The number of bees from $B$ assigned to each cell in $C$ is proportional to the relative amount of net nectar in the cell. The nectar in those cells will be depleted accordingly. We use an average of $40 \mathrm{mg}$ of gross nectar collected per bee per trip [Beekman and Lew, 2008].

\section{INITIAL SIMULATION EXPERIMENTS}

This section includes some initial simulation experiments, where the simulator was used with a configuration consisting of an area of $580 \mathrm{~km}^{2}(29 \mathrm{~km}$ by $20 \mathrm{~km})$ using a resolution of 50 by 35 cells $\left(0.3 \mathrm{~km}^{2}\right.$ 


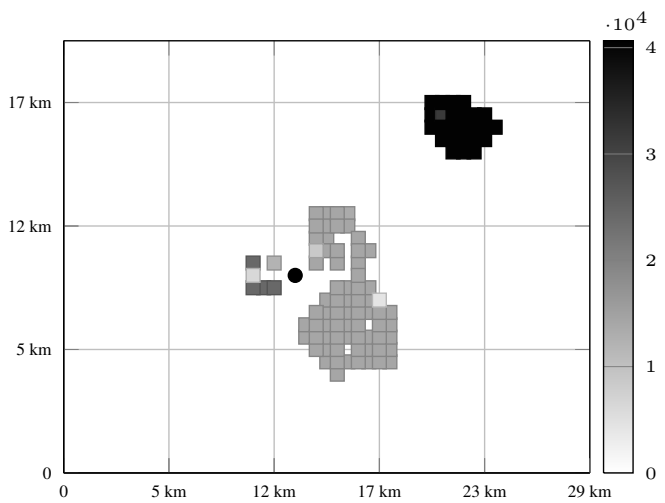

(a) Remaining net nectar (mg) after $1^{\text {st }}$ foraging trip.

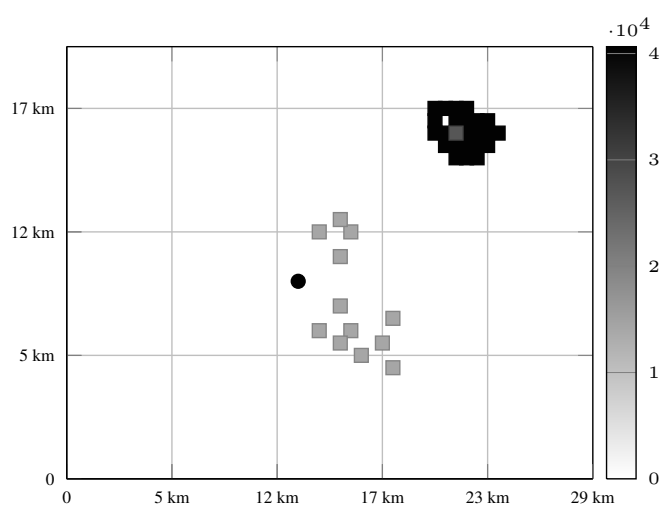

(c) Remaining net nectar (mg) after $6^{\text {th }}$ foraging trip.

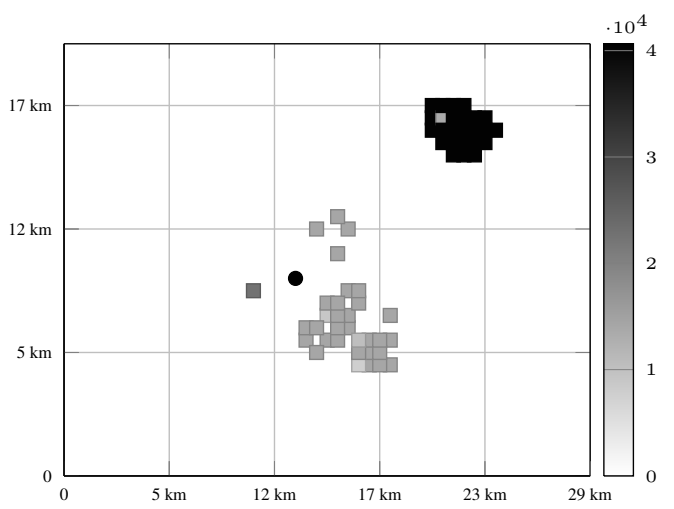

(b) Remaining net nectar (mg) after $3^{\text {rd }}$ foraging trip.

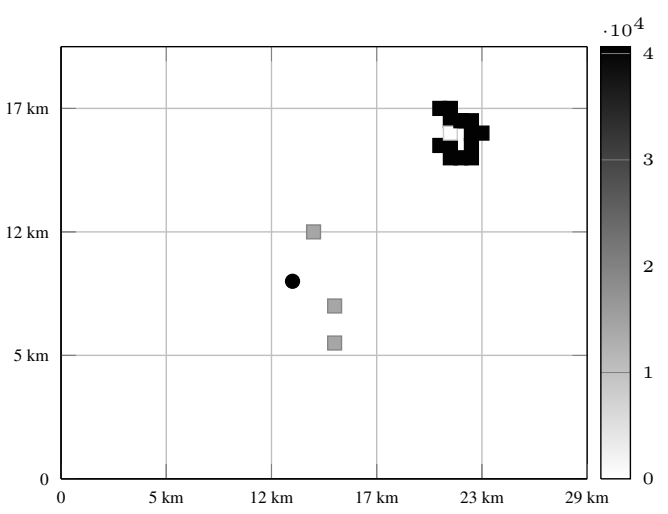

(d) Remaining net nectar (mg) after $9^{\text {th }}$ foraging trip.

Figure 3: Nectar depletion over four subsequent foraging trips during the same day by bees foraging in three different flower patches. Dot in the centre represents the beehive. Squares represent a flower patch section with the darker the colour the higher the amount of net nectar left.

per cell), with a single beehive of 15000 foraging bees at the centre, surrounded by the following 3 flower patches with only one season each: i) $31 \mathrm{~km}^{2}$ to the east containing 10000 flowers with $80 \mathrm{mg}$ with nectar of 0.8 quality rate; ii) $2 \mathrm{~km}^{2}$ to the west containing 500 flowers with $200 \mathrm{mg}$ with nectar of 0.7 quality rate; and iii) $8 \mathrm{~km}^{2}$ to the north-east containing 5000 flowers with $100 \mathrm{mg}$ of 1 quality rate.

Figure 3 offers a graphical view of how nectar is depleted along alternate foraging trips in an arbitrary day where the average temperature was $25^{\circ} \mathrm{C}$ with 12 hours of daylight, yielding 9 daily foraging trips. These results show how bees will prefer to forage at closer distances as long as there is nectar available. It also makes clear how bees will select food sources according to their net nectar (i.e. quality and quantity). In consequence, the northern flower patch containing the best nectar is depleted sooner than the other two.

While the results are interesting and appear to concur with experience-based expectations, a scientific evaluation of the accuracy of the overall predictions of the simulator will await future work using actual observations.

\section{CONCLUSIONS}

This work presents a simulator of honeybee colony foraging behavior. In contrast to existing work, we have designed a simulator suitable for covering long time periods and simulating honeybee responses to local flora resources. The simulator has convenience and power that comes from being Web based. Its simulation model stochastic and discrete in time. It is also explanatory, using well-established entomological knowledge encapsulated in four interrelated models. It can support any number of beehives and floral patches over a two dimensional landscape of unrestricted size and user-selected resolution. 
In some follow-on work we will investigate ways to evaluate the accuracy and realism of the simulator using actual observations of bee foraging. We will also extend the model to take account of the fact that honeybees also forage for pollen as well as nectar and the mechanics are distinct. This is important for applications to horticultural pests such as Fire Blight.

\section{REFERENCES}

Aken, J. V. (1984). An Efficient Ellipse-Drawing Algorithm. IEEE Computer Graphics and Applications, $4: 24-35$.

Bailis, P., Nagpal, R., and Werfel, J. (2010). Positional Communication and Private Information in Honeybee Foraging Models. In Proceedings of the 7th international conference on Swarm intelligence, pages 263-274, Berlin, Heidelberg. Springer-Verlag.

Beekman, M. and Lew, J. B. (2008). Foraging in Honeybees-When Does it Pay to Dance? Behavioral Ecology, 19(2):255-261.

Camazine, S. and Sneyd, J. (1991). A Model of Collective Nectar Source Selection by Honey Bees: Self-organization Through Simple Rules. Journal of Theoretical Biology, 149(4):547-571.

Clark, J., Macklin, E., and Wood, L. (1998). Stages and Spatial Scales of Recruitment Limitation in Southern Appalachian Forests. Ecological Monographs, 68(2):213-235.

de Vries, H. and Biesmeijer, J. C. (1998). Modelling Collective Foraging by Means of Individual Behaviour Rules in Honey-bees. Behav Ecol Sociobiol, 44:109-124.

García Adeva, J., Sousa-Majer, M., Botha, J., Hanbury, C., Hardie, D., and Reynolds, M. (2010). Modelling the Establishment and Spread of Emergency Plant Pests (EPPs) in Australia: Simulate or Suffer. In Global Biosecurity Conference.

Ghosh, S. and Marshall, I. (2005). Simple model of Collective Decision Making during Nectar Source selection by Honey Bees. In Workshop on Memory and Learning Mechanisms in Autonomous Robots (ECAL 2005), page 10.

Haines, E. (1994). Graphics Gems IV, chapter Point in Polygon Strategies. Morgan Kaufmann.

Law, A. M. and Kelton, W. D. (1991). Simulation Modeling and Analysis. McGraw-Hill, New York, NY, second edition.

Moosbeckhofer, R., Loncaric, I., Oberlerchner, J., Persen, U., Ertl, C., and Donat, C. (2008). Use of Honeybees (Apis mellifera) as Vectors for Fire Blight Antagonists in Field Experiments. In Acta Horticulturae 793: XI International Workshop on Fire Blight, pages 461-465.

Morse, R. and Calderone, N. (2000). The Value of Honey Bees as Pollinators of U.S. Crops in 2000. Bee Culture Magazine, (128):1-14.

Odum, E. (1971). Fundamentals of Ecology. W.B. Saunders Company, Philadelphia.

Pitt, J. P. W. (2008). Modelling the Spread of Invasive Species Across Heterogeneous Landscapes. PhD thesis.

Potts, S., Biesmeijer, J., Kremen, C., Neumann, P., Schweiger, O., and Kunin, W. (2010). Global Pollinator Declines: Trends, Impacts and Drivers. Trends in Ecology and Evolution, 25(6):345-353.

Seeley, T. D. (1985). Honeybee Ecology: a Study of Adoptation in Social Life. Princeton Univ. Press, Princeton, NJ.

Seeley, T. D., Camazine, S., and Sneyd, J. (1991). Collective Decision-making in Honey Bees: How Colonies Choose Among Nectar Sources. Behavioral Ecology and Sociobiology, 28(4):277-290.

Shaw, M. W. (1995). Simulation of Population Expansion and Spatial Pattern when Individual Dispersal Distributions do not Decline Exponentially with Distance. Proceedings of the Royal Society London Series B, 259:243-248.

Swannack, T. (2008). Ecological Modeling: A Common-Sense Approach to Theory and Practice. WileyBlackwell.

Winston, M. (1991). The Biology of the Honey Bee. Harvard University Press. 